\title{
Artículo \\ La evaluación del desempeño docente en la educación pública salvadoreña
}

\author{
Assessment of the teacher's performance \\ in the Salvadoran public school
}

Óscar Martínez Peñate*

ompenate@yahoo.com

ISSN 1996-1642, Editorial Universidad Don Bosco, año 10, No.18, julio-diciembre de 2016, pp. 29-46 Recibido: 12 abril de 2016. Aprobado: 30 de junio de 2016

\begin{abstract}
Resumen
El propósito de esta investigación fue comprender la importancia de la evaluación del desempeño docente y la incidencia en la calidad de la educación. Para su abordaje teórico se recurrió a Pierre Bourdieu, y para su abordaje metodológico se utilizó la entrevista semiestructurada aplicada a funcionarios del Ministerio de Educación de El Salvador, profesores y directores de centros educativos públicos y privados. También se utilizaron grupos focales con estudiantes universitarios de nuevo ingreso. Se realizó el análisis estadístico descriptivo de las variables "calidad docente" y "rendimiento académico estudiantil".
\end{abstract}

Palabras clave: desempeño docente, educación, evaluación docente, profesorado, sistema educativo.

\begin{abstract}
The aim of this study was to understand the importance of assessment of the teacher's performance and its impact on the quality of education. The theoretical support of the study was based on the ideas of Pierre Bourdieu and the methodological approach included semi structured interviews applied to the Ministry of Education officials, teachers and school principals from private and public schools. Freshmen from several universities were also interviewed through focus groups. Results were also gathered though descriptive statistic analysis of both variables teaching quality and academic performance.
\end{abstract}

Keywords: teacher performance, education, teacher assessment, faculty, educational system.

\footnotetext{
* El autor es Máster en Ciencia Política de la Université du Québec à Montréal (UQAM), Canadá y Doctorando en Ciencias Sociales Universidad de El Salvador, El Salvador. Es investigador académico de la Escuela de Formación Registral (ESFOR) del Centro Nacional de Registro (CNR) (El Salvador).
} 


\section{Introducción}

En El Salvador no existe bibliografía nacional sobre este tema. No obstante esta limitante, la investigación se realizó para conocer si la evaluación del desempeño docente del sistema educativo público. Se espera que sus resultados se conviertan en un insumo de importancia que puede conducir entre otras cosas a realizar modificaciones, reformas o cambios estructurales en lo curricular con la creación de nuevos programas de estudio; en la reconfiguración del pensum de formación de docentes; o tomar medidas para el mejoramiento del clima escolar que beneficie las interacciones sociales entre estudiantes, profesores, directores y padres de familia.

El Ministerio de Educación no evalúa el desempeño docente. Es decir, no la hacen los asesores pedagógicos, supervisores e inspectores y tampoco otras autoridades docentes. Tampoco es realizada por padres de familia, pares, organismos internacionales ni se hace la autoevaluación.

La evaluación realizada por estudiantes al desempeño docente se traduce en uno de los parámetros a tomar en consideración para determinar la calidad del sistema educativo. Pero esos resultados no se pueden adoptar como concluyentes ni utilizarlos para realizar generalizaciones. La evaluación del desempeño docente, cuando es enfocada en razón epistemológica desde diferentes ángulos, permite tener una percepción amplia del contexto.

Con la encuesta realizada en el 2013, a pesar de que se denomina Evaluación de la calidad del sistema educativo público por estudiantes de educación media, en realidad no constituye una evaluación del sistema educativo público, tan solo es una parte. No obstante, refleja en cierta medida alguna calidad, si se toma en consideración que la educación media es la culminación de un proceso de once años de estudio. Además, no refleja la calidad del sistema educativo salvadoreño, ya que si hubiera calidad en la educación los estudiantes, al efectuar la Prueba de Aprendizaje y Aptitudes para Egresados de Educación Media (PAES), saldrían mejor evaluados.

\section{Abordaje teórico de la evaluación del desempeño docente}

Tomaremos como punto de análisis algunos elementos teóricos abordados por Pierre Bourdieu sobre la educación, en particular aquellos que tengan relación con el desempeño docente.

Bourdieu ha sido uno de los intelectuales contemporáneos que mostró interés en que la educación fuera la base fundamental de cohesión y desarrollo social, de crecimiento económico, avances científicos e innovación tecnológica. Los aportes teóricos de Bourdieu fueron importantes en los cambios al sistema francés de educación. Sin embargo, su contribución intelectual sobrepasó las

La evaluación del desempeño docente en la educación pública 30. salvadoreña frontera gala y europea, y ahora es un referente obligatorio en el estudio de la educación. 
De acuerdo con Bourdieu, en el buen desempeño docente intervienen directa e indirectamente varios factores que incidirán en la calidad del sistema educativo. El desempeño docente es solamente una variable en el estudio del sistema educativo. Entre los aportes de Bourdieu, podemos mencionar los contenidos de la educación, lo cual conlleva un análisis de los saberes comprendidos en los programas de estudio con el objetivo de lograr la coherencia, la unidad y la concreción de los mismos. En la medida que los contenidos programáticos sean generales y abstractos se produce la incomprensión y la incoherencia. Los temas o ítems vagos dan lugar a interpretaciones laxas. Por lo que los programas de estudio ameritan ser revisados frecuentemente para actualizarlos al introducirle datos, aportados por el avance científico y tecnológico en las diferentes disciplinas científicas, sin menoscabo de la importancia del conocimiento y de la evolución histórica de la humanidad y sus creaciones.

Pierre Bourdieu en su libro Capital cultural, escuela y espacio social (1997, p. 62), explica que la evaluación del estudiante no es del conocimiento en sí, sino la utilización que el estudiante haga en espacios diversos en los que le fue enseñado. Es decir, el uso de los conocimientos, "que permitan evaluar la capacidad inventiva, el sentido crítico y el sentido práctico". Cuando Bourdieu aborda la importancia de la forma de transmitir el conocimiento por el docente, no se refiere únicamente a la utilización de las nuevas tecnologías de la comunicación, sino sobre todo, a la asimilación reflexiva y crítica de los modos de pensar (deductivo, experimental e histórico). Es decir, la no reificación de las nuevas tecnologías en detrimento del racionamiento y la reflexión crítica. Es sentar las bases de la formación científica y la creación de ciudadanos capaces de resolver problemas y dar el paso más allá de la crítica.

Bourdieu considera vana la formación enciclopedista, enseñar una gran cantidad de asignaturas y tratar de explicar lo más que se pueda de cada una de ellas; se privilegia las asignaturas básicas y dar el conocimiento más importante de forma concisa.

Los conceptos fundamentales según la teoría de Pierre Bourdieu, son el habitus', campo social ${ }^{2}$ y capital. En este orden, el trabajo pedagógico es la formación del habitus a través del conocimiento que se desprende de los contenidos de los programas de estudio, es la interiorización dosificada del entorno, que va a durar más allá del proceso enseñanza-aprendizaje, porque es un proceso de socialización que finaliza con la muerte. Bourdieu y Passeron le denominan "la interiorización de los principios de una arbitrariedad cultural" (1977, p. 9).

Cada estudiante que ingresa al sistema educativo tiene un habitus primario formado en el seno familiar, y este corresponde directamente a su procedencia

1. El habitus viene a ser la interiorización del ser humano de las estructuras sociales, lo que en cierta medida lo influenciará para mantener y reproducir las estructuras externas, en otras palabras es la conversión de lo objetivo en subjetivo, a través de un proceso mental.

2. El espacio social es el campo donde se ponen en práctica el habitus, es el lugar donde se establecen las interacciones sociales y se evidencian las diferencias en cuanto a posiciones sociales, que se desprenden de los estatus sociales basados en el poder y la autoridad, que se derivan del capital económico, cultural y social. 
social, por tanto la eficacia del trabajo pedagógico del docente para inculcar el habitus que se pretende enseñar, está en relación con la distancia del habitus de origen social del estudiante.

El rendimiento académico de los estudiantes también tiene relación con el capital cultural que la familia le ha inculcado al joven. Los estudiantes que no poseen un capital cultural muy elevado, tendrán dificultades en cuanto a los logros de aprendizaje. El rendimiento escolar también tiene relación con el origen social del estudiante ya que los jóvenes que provienen de la clase media o alta tienen mayores posibilidades de éxito. Esto significa que no tiene que ver solo con las actitudes individuales del educando, sino que el peso del capital cultural también tiene relación con el capital económico. Es lo que se llama la reproducción del estatus quo (Gutiérrez, 2003, p. 116).

La institución educativa instruye en el dominio de los conocimiento y debe de hacerlo de la mejor forma e ir más allá; no es la única responsable de la educación y de la socialización del estudiante sino que es complementario con la que recibe de sus padres.

El habitus secundario es el formado fuera del entorno familiar, según Bourdieu, "la combinación del trabajo pedagógico primario con otras formas secundarias del mismo implica procesos de aculturación, deculturación y reculturación" (Penagos, 2009, p. 15). El habitus es la representación o el reflejo de las condiciones sociales, económicas, políticas y culturales de la sociedad, es la manifestación de las características específicas, consiste en la internalización de las estructuras socioeconómicas; el estudiante lo que hace es reproducir los usos y prácticas sociales en relación al campo que pertenece (Zúñiga, 2009, p. 157).

Como ejemplo se puede mencionar que el sistema educativo costarricense y el salvadoreño, tienen diferencias obviamente en lo social y cultural, etc., ambos sistemas forman ciudadanos con perfiles delineados, entre otros, por el sistema educativo nacional para mantener y reproducir el habitus formado en el país respectivo. Sin embargo, son dos formas distintas de percibir la realidad nacional y de establecer las relaciones interpersonales. Asimismo, son dos formas diferentes de relación que tienen los ciudadanos con las instituciones estatales. Según el legado de Bourdieu; "La forma en que los individuos perciben el mundo está ligado a su posición en el mundo social" (Marín, 2010, p. 75).

La función del sistema educativo nacional es reproducir las estructuras económicas, sociales y culturales. En sentido inverso, se podría afirmar que el sistema educativo es el reflejo de las estructuras económicas, sociales y culturales imperantes en la sociedad. El nivel del sistema educativo tendrá relación directa con el de los principales líderes y sus respectivas instituciones protagónicos del país, en los ámbitos de la realidad nacional y el nivel de las estructuras societales.

La evaluación del desempeño docente en la educación pública

32. salvadoreña
Esta acción pedagógica es una arbitrariedad porque es una imposición de un grupo social o de una élite económica la que impone a los estudiantes a través del sistema educativo nacional, la reproducción de las estructuras dominadas 
desde su posición de dominante. Las instituciones educativas son instrumentos de dominación y reproducción porque inculcan una cultura dominante, la reproducción de las relaciones de clase y la función ideológica de encubrir la parcialidad, la injusticia y la inequidad y mostrar la apariencia de neutrales e independientes (Sánchez, 2002, p. 5).

Esta situación de imposición y reproducción que se da en el sistema educativo constituye violencia simbólica, al imponer el sistema de simbología y de significados del grupo o de clase dominante a los hijos de la clase dominada, considerado como legítimo, normal y hasta natural, la base fundamental de la violencia simbólica la ejerce el docente a través de la acción pedagógica. El sistema educativo contribuye de esta forma a la reproducción de las desigualdades sociales legitimándolas.

El Estado, en este proceso juega un papel importante porque el grupo o clase social que lo controle, no necesitará la fuerza para imponer la dominación sobre las demás clases sociales. Así, el Estado apoyado en la institución educativa y la familia estarán sintonizados en la misma onda de frecuencia, para imponer pacíficamente su habitus (Santos, 2011, p. 11).

Bourdieu sostenía que esta dominación es más estable y duradera que la dominación fundada solamente en el capital económico, como es la alienación y el consumismo. Asimismo, explicaba que la escuela es un espacio donde se fabrican estudiantes con una forma de pensar y actuar frente a diferentes situaciones y campos.

Idealmente, la educación es un medio para lograr la movilidad social, disminuir la brecha de la desigualdad social y de la inequidad económica, para construir, reconstruir o consolidar la democracia (Starkie, 2006, p. 435). Esto significa que los contenidos en los programas de estudio y la metodología pedagógica tienen relación directa con el perfil delineado del estudiante; es decir, el perfil influye en la elaboración de los programas de estudio y en la acción pedagógica. Los programas son una guía para los docentes y los estudiantes, no constituyen una camisa de fuerza, contienen objetivos en donde explican lo que se persigue y cuál es la orientación filosófica.

La formación de alto nivel de los maestros ${ }^{3}$ y la capacitación obligatoria permanente son elementos de análisis en la evaluación del desempeño docente. La aprobación de varias pruebas para establecer la capacidad y la aptitud de los profesores así como la contratación por oposición coadyuvaría a la cualificación del cuerpo docente del sistema educativo nacional. De la misma manera, la realización periódica de la evaluación del desempeño docente realizado por padres, autoridades académicas, pares, autoevaluación y estudiantes mejoraría también su desempeño.

3. Tiene relación directa con elevar: "Los parámetros para el ingreso a los programas de educación docente; la calidad de los instituciones de educación docente; y los parámetros de contratación de los nuevos profesores" (Barbara Bruns \& Luque, 2014, p. 25) 


\section{Materiales y métodos La recolección de los datos}

Se realizó una búsqueda bibliográfica en los repositorios virtuales de las bibliotecas de las universidades salvadoreñas donde se imparte la carrera de profesorado, así como en la biblioteca de la Escuela Superior de Maestros (ESMA) del Ministerio de Educación de El Salvador. Además, se efectuó una búsqueda por Internet en las principales bases de datos, en revistas especializadas en educación, libros, tesis y documentos que abordaran el tema de la evaluación docente.

Se realizaron entrevistas semiestructuradas sobre la evaluación del desempeño docente a profesores de educación media del sector público y privado. De igual forma, se entrevistó al Ministro de Educación y a los principales funcionarios del Ministerio de Educación de El Salvador.

Se organizaron grupos focales con estudiantes universitarios de nuevo ingreso provenientes del sector público y privado, se consultó a académicos especialistas en educación.

Se realizó el análisis estadístico descriptivo de las variables "calidad docente" y "rendimiento académico estudiantil", se retomaron los resultados de la encuesta: "Evaluación de la calidad del sistema educativo público por estudiantes de educación media". Realizado por el Centro de Opinión Pública de la Universidad Francisco Gavidia en 2013, el trabajo de campo se efectuó del 27 al 31 de mayo de 2013.

La encuesta se realizó con la utilización de un cuestionario individual preestructurado, a 1,006 estudiantes de educación media del sector público de educación, distribuidos en 44 institutos nacionales, con un muestreo probabilístico con afijación proporcional al tamaño de la población de cada instituto, con un nivel de confianza del $95 \%$ y un error muestral de \pm 2.5 .

La población estudiantil de 44 institutos nacionales es la parte de la población de la cual se tomó la muestra de 1,006 estudiantes:

\section{La calidad docente}

La variable "calidad docente" se analizó a través de los siguientes indicadores:

1. Método pedagógico

2. Aclaración de las dudas que los estudiantes tienen sobre la asignatura.

3. Cumplimiento del calendario escolar.

4. Promoción del auto-aprendizaje y la investigación en la asignatura.

5. Procedimientos disciplinarios utilizados para alumnos problemáticos.

6. Procedimientos e instrumentos para evaluar el aprendizaje de los estudiantes.

7. Uso de la tecnología en el desarrollo de las clases.

8. Comprensión, por parte de los estudiantes, del lenguaje utilizado por el docente en el desarrollo de las clases.

evaluación del desempeño docente en la educación pública 34. salvadoreña 
Cada indicador se expresó como una pregunta, la cual tiene varias opciones de respuesta, entre las cuales, el estudiante eligió alguna. Los resultados obtenidos de la medición de los 9 indicadores antes mencionados, se representaron en tablas, diagramas circulares y gráficos de barras. A partir de los mismos se analizó el resultado de cada opción de respuesta, se comparó entre ellas para ver en cuál se ubicó la mayoría de estudiantes.

\section{El rendimiento académico del estudiante}

La variable "rendimiento académico estudiantil" se analizó a través de los siguientes indicadores:

1. Promedio de notas.

2. Preparación para la PAES de parte de la institución educativa.

3. Tiempo dedicado al estudio y tareas.

El promedio de notas fue evaluado en forma de pregunta: ¿Cuál es tu promedio de notas en cada área?, para las cuatro asignaturas básicas: Matemática, Ciencias Naturales, Sociales y Letras. Se calculó para cada asignatura la media, mediana, moda, desviación típica, varianza, rango, valor máximo y mínimo. Se utilizó el programa estadístico SPSS.

Los indicadores: Preparación para la PAES de parte de la institución educativa y Tiempo dedicado al estudio y tareas, se analizaron mediante una tabla de frecuencias y porcentajes relativos, se observó cuál opción tuvo el porcentaje más alto.

Se observó cómo se relacionaron entre sí algunos indicadores, mediante el cruce entre los mismos, utilizando tablas de contingencia. Se analizó si el promedio de notas depende de la preparación para la PAES de parte de la institución educativa y si depende del tiempo que el estudiante dedica al estudio. Se evaluó si existe una dependencia entre los indicadores mediante el análisis de hipótesis a través de la prueba chi-cuadrado. Se utilizó el programa estadístico SPSS para la realización de este análisis.

Se realizó el cruce de los indicadores siguientes:

1. Promedio de notas vs. Preparación para la PAES de parte de la institución educativa.

2. Promedio de notas vs Tiempo dedicado al estudio y tareas.

3. Se planteó una hipótesis general la cual se operacionalizó con dos hipótesis de trabajo y sus respectivas hipótesis nulas, las cuales relacionan los indicadores mencionados. Los mismos se escogieron porque se consideró que el análisis de la relación entre esos indicadores contribuye a la comprobación de la hipótesis general.

\section{Resultados}

Se realizó el análisis estadístico descriptivo de las variables "calidad docente" y "rendimiento académico estudiantil", retomando los resultados de la encuesta: 
"Evaluación de la calidad del sistema educativo público por estudiantes de educación media". Realizado por el Centro de Opinión Pública de la Universidad Francisco Gavidia en 2013.

El estudio desarrollado es de tipo cuantitativo. La encuesta se realizó, utilizando un cuestionario individual pre-estructurado, a 1006 estudiantes de educación media del sector público de educación, distribuidos en 44 institutos nacionales, con un muestreo probabilístico con afijación proporcional al tamaño de la población de cada instituto, con un nivel de confianza del $95 \%$ y un error muestral de \pm 2.5 .

\section{Indicadores de la variable calidad docente}

Los indicadores considerados para medir la variable "calidad docente", nueve en total, se enumeran a continuación junto con los resultados obtenidos a partir de la medición de cada indicador:

1. Método pedagógico: El $42.4 \%$ de los estudiantes encuestados consideraron que hay buen nivel de participación estudiantil en la clase, el 32.5\% consideró que los docentes los motivan a que lean, razonen y analicen; mientras que el $27.1 \%$ indicó que se utiliza el dictado y el uso de la pizarra, el 14\% manifestó que copian de los libros y $6 \%$ indicó que utilizan los laboratorios.

2. Aclaración de las dudas que los estudiantes tienen sobre la asignatura: El $81 \%$ de los estudiantes consideran que los docentes aclaran sus dudas sobre la asignatura, el $11 \%$ indican que no lo hacen y el $8 \%$ es indiferente.

3. Cumplimiento del calendario escolar: El $50 \%$ de los estudiantes consideran que el calendario escolar se cumple en su totalidad. El resto considera que se cumple parcialmente.

4. Promoción del auto-aprendizaje y la investigación en la asignatura: La mayoría de los estudiantes (85\%) están de acuerdo en que los profesores promueven el auto-aprendizaje e investigación. Los resultados permiten inferir que los docentes están aplicando el constructivismo, en el cual, los estudiantes son los constructores de su propio aprendizaje.

5. Procedimientos disciplinarios utilizados para alumnos problemáticos: La mayoría de docentes (44\%) prefieren remitir a los estudiantes problemáticos a la dirección del centro escolar, los sacan del aula (27\%), los mandan a hacer tareas de limpieza (18\%), los expulsan.

6. Procedimientos e instrumentos para evaluar el aprendizaje de los estudiantes: La mayoría de estudiantes (85\%) consideran que los profesores utilizan diferentes procedimientos e instrumentos para evaluarlos. Se puede inferir que los docentes utilizan un sistema adecuado para la evaluación de los aprendizajes.

7. Uso de la tecnología en el desarrollo de las clases: La mayoría de docentes (66\%) utilizan la tecnología en el desarrollo de las clases. Se infiere que la mayoría de los docentes conocen sobre las tecnologías de la información.

8. Comprensión de los estudiantes del lenguaje utilizado por el docente en el desarrollo de las clases: De acuerdo al gráfico 1 la mayoría de estudiantes (92\%) nunca o rara vez comprende el lenguaje utilizado por los docentes durante el desarrollo de las clases. 
Gráfico 1. Comprensión de los estudiantes, del lenguaje utilizado por el docente en el desarrollo de las clases.

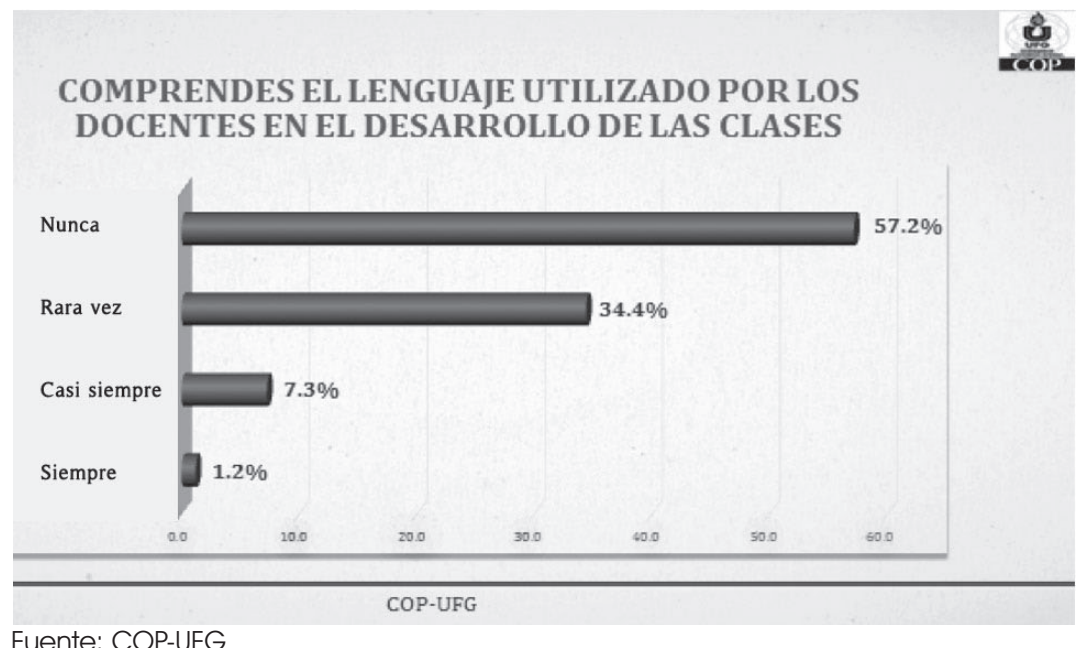

9. Frecuencia en la comunicación del docente con los padres de familia. Se observa en el gráfico 2, que la mayoría de docentes (57\%) siempre y casi siempre, se comunican frecuentemente con los padres de familia.

Gráfico 2. Frecuencia en la comunicación del docente con los padres de familia.

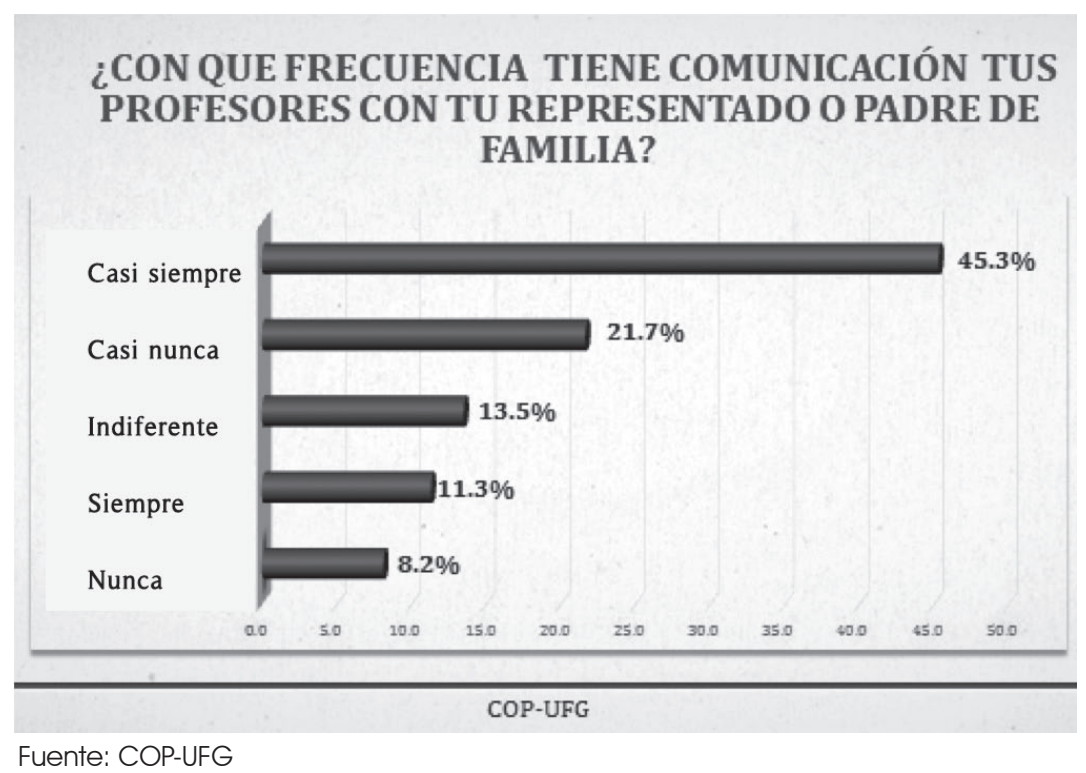

Indicadores de la variable rendimiento académico estudiantil

Los indicadores considerados para medir la variable rendimiento académico estudiantil se enumeran y se presentan a continuación con sus resultados:

1. Promedio de notas

Análisis de los datos del cuadro 1 
En el cuadro 3 se muestran los resultados correspondientes a la pregunta: ¿Cuál es tu promedio de notas en cada área?

Los resultados indican que Matemática es la asignatura con la nota promedio más baja y Letras obtuvo la nota más alta. Los resultados muestran que el aprendizaje de la Matemática es deficiente en relación con el resto de asignaturas. La causa de esta deficiencia posiblemente sea porque los estudiantes no han logrado desarrollar el pensamiento lógico-matemático, debido a que los docentes no emplean una metodología didáctica adecuada para la enseñanza de la Matemática.

En las asignaturas de Ciencias Naturales, Sociales y Letras obtienen un mejor promedio posiblemente porque el aprendizaje se basa en la memorización de datos.

Las desviaciones típicas obtenidas indican que las notas promedio de las cuatro asignaturas, son representativas de la muestra.

Los valores de las medianas, para las cuatro asignaturas, están en el rango [7 - 8], las cuales representan respectivamente, la nota promedio ubicada en el centro de la distribución de datos. Para el caso de Matemática la mediana es 7.0, por tanto, el $50 \%$ de notas son inferiores a este valor y el restante $50 \%$ superior al mismo. La asignatura Letras tiene una mediana de 8.0, siendo la nota en el centro de la distribución e indica que el $50 \%$ de las notas es superior a dicho valor y el otro $50 \%$ es inferior. Además, Letras tiene la mayor mediana en relación con las demás asignaturas, reflejando un mayor rendimiento académico.

La moda o valor que más se repite es igual para las cuatro asignaturas. La nota promedio de 7.0 es la que más se repite, indicando que los estudiantes no logran diferenciar su rendimiento en las diferentes asignaturas consideradas.

El percentil 25 varía en el rango [6.0 - 7.0], e indica que el 25\% de las notas son inferiores a los valores del rango. En matemática el $25 \%$ de notas es inferior a 6.0, en Ciencias Naturales, Sociales y Letras el $25 \%$ de notas es inferior a 7.0. Matemática obtiene el menor valor en el percentil considerado.

El percentil 50 varía en el rango [7.0 - 8.0], e indica que el 50\% de las notas son inferiores a los valores del rango. En este caso la mitad de los alumnos manifestó que tiene una nota promedio inferior a 7.0 en Matemática y Ciencias Naturales, inferior a 7.5 en Sociales e inferior a 8.0 en Letras. Matemática y Ciencias Naturales obtienen el menor valor en el percentil 50.

El percentil 75 varía en el rango de valores [8.0 - 8.4]. Para Matemática, Ciencias Naturales y Sociales el $75 \%$ de notas es inferior a 8.0 y para Letras es inferior a

La evaluación del desempeño docente en la educación pública

38. salvadoreña
8.4. Matemática, Ciencias Naturales y Sociales obtienen el menor valor en el percentil 75 . 
En base al análisis de los percentiles se observa que los estudiantes tienen promedios de notas en las asignaturas, en el orden de menor a mayor, siguiente: Matemática, Ciencias Naturales, Sociales y Letras. De acuerdo a lo anterior se interpreta que el grado de dificultad, de menor a mayor, en las asignaturas es: Letras, Sociales, Ciencias Naturales y Matemática.

Los datos perdidos se deben a que los estudiantes no seleccionaron alguna respuesta, o que los estudiantes seleccionaron más de una respuesta.

En resumen, la similitud entre las medidas de tendencia central en las cuatro asignaturas, se debe a las valoraciones semejantes que hacen los estudiantes con respecto a las notas que obtienen en dichas asignaturas. Indica que no saben diferenciar el rendimiento académico que tienen en cada asignatura.

Los datos del cuadro 3 no reflejan el rendimiento académico real de los estudiantes, porque no se basan en notas obtenidas como resultado de evaluaciones objetivas. Son el producto de la valoración personal de los alumnos.

Cuadro 1. Estadísticos para cuatro asignaturas. ¿Cuál es tu promedio de notas en cada área?

\begin{tabular}{|c|c|c|c|c|c|}
\hline & & Matemáticas & $\begin{array}{l}\text { Ciencias } \\
\text { Naturales }\end{array}$ & $\begin{array}{l}\text { Ciencias } \\
\text { Sociales }\end{array}$ & Letras \\
\hline & $\begin{array}{l}\text { Válidos } \\
\text { Perdidos }\end{array}$ & $\begin{array}{c}986 \\
20\end{array}$ & $\begin{array}{c}983 \\
23\end{array}$ & $\begin{array}{c}984 \\
22\end{array}$ & $\begin{array}{c}968 \\
38\end{array}$ \\
\hline Media & & 7.0739 & 7.3973 & 7.4800 & 7.5748 \\
\hline Mediana & & 7.0000 & 7.0000 & 7.5000 & 8.0000 \\
\hline Moda & & 7.00 & 7.00 & 7.00 & 7.00 \\
\hline Desv. típ. & & 1.10961 & 1.17850 & 1.18657 & 1.26082 \\
\hline Varianza & & 1.231 & 1.389 & 1.408 & 1.590 \\
\hline Rango & & 7.00 & 7.00 & 7.00 & 7.00 \\
\hline Mínimo & & 3.00 & 3.00 & 3.00 & 3.00 \\
\hline \multirow[t]{2}{*}{ Máximo } & & 10.00 & 10.00 & 10.00 & 10.00 \\
\hline & 25 & 6.0000 & 7.0000 & 7.0000 & 7.0000 \\
\hline \multirow[t]{2}{*}{ Perceptiles } & 50 & 7.0000 & 7.0000 & 7.5000 & 8.0000 \\
\hline & 75 & 8.0000 & 8.0000 & 8.0000 & 8.4000 \\
\hline
\end{tabular}

Fuente: elaboración propia a partir de la base de datos del estudio: "Evaluación de la calidad del sistema educativo público por estudiantes de educación media". Realizado por el Centro de Opinión Pública, Universidad Francisco Gavidia, 2013.

2. Preparación para la PAES de parte de la institución educativa La mayoría de los estudiantes (54\%) consideran que su institución educativa los prepara muy bien para la PAES, el $8 \%$ considera que no los prepara.

3. Tiempo dedicado al estudio y tareas 
La mayoría de estudiantes (60\%) dedican entre 1 a 3 horas diarias al estudio. El $25 \%$ estudia menos de 1 hora, el $12 \%$ entre 3 y 4 horas y el $3 \%$ de estudiantes dedica más de 4 horas a estudiar.

Cruce de indicadores (tablas de contingencia)

Con el objetivo de observar cómo se relacionan entre sí algunos indicadores, se realizó el cruce entre los mismos mediante las tablas de contingencia.

Se realizó el cruce de los indicadores siguientes:

2.0 Promedio de notas vs. Preparación para la PAES de parte de la institución educativa.

3.0. Promedio de notas vs Tiempo dedicado al estudio y tareas.

Se evaluó si existe una relación entre los indicadores, mediante el análisis de hipótesis a través de la prueba chi-cuadrado.

\section{Sistema de hipótesis}

Se planteó una hipótesis general la cual se operacionalizó con dos hipótesis de trabajo y sus respectivas hipótesis nulas, las cuales relacionan los indicadores específicos: promedio de notas versus Preparación para la PAES de parte de la institución educativa y promedio de notas versus Tiempo dedicado al estudio y tareas. Dichos indicadores se escogieron porque se consideró que el análisis de la relación entre los mismos contribuye a la comprobación de la hipótesis general.

Hipótesis general:

La encuesta de la evaluación de la calidad del sistema educativo público por estudiantes de educación media, no refleja la calidad del sistema educativo salvadoreño, si hubiera calidad en la educación los estudiantes al efectuar la PAES saldría mejor evaluados.

1. Conjunto de hipótesis que relacionan los indicadores: Promedio de notas vs preparación para la PAES de parte de la institución educativa".

Hipótesis de trabajo $\left(\mathrm{Hi}_{1}\right)$ :

"El promedio de notas en cada asignatura depende de la preparación para la PAES de parte de la institución educativa".

Hipótesis nula $\left(\mathrm{Ho}_{1}\right)$ :

"El promedio de notas en cada asignatura no depende de la preparación para la PAES de parte de la institución educativa".

2. Conjunto de hipótesis que relacionan los indicadores: Promedio de notas vs Tiempo dedicado al estudio y tareas.

Hipótesis de trabajo $\left(\mathrm{Hi}_{2}\right)$ :

"El promedio de notas en cada asignatura depende del tiempo dedicado al estudio y tareas".

Hipótesis nula $\left(\mathrm{HO}_{2}\right)$ :

"El promedio de notas en cada asignatura no depende del tiempo dedicado al estudio y tareas". 
Análisis de los indicadores: Promedio de notas vs. Preparación para la PAES de parte de la institución educativa.

Tabla de contingencia

Al analizar el cuadro 2 se observa que la mayoría de los estudiantes considera que su institución educativa les prepara muy bien para la PAES. Además, se observa que los porcentajes más altos (encerrados en un círculo) corresponden a los rangos de notas más bajas. Significa que los estudiantes con notas bajas reconocen que la institución los prepara adecuadamente para la PAES. Sin embargo, posiblemente las notas bajas son el resultado de la falta de esfuerzo personal.

Cuadro 2. Promedio de notas vs Preparación para la PAES de parte de la institución educativa.

\begin{tabular}{|c|c|c|c|c|}
\hline \multicolumn{5}{|c|}{ RENDIMIENTO Y PREPARACIÓN PARA LA PAES } \\
\hline \multirow[t]{2}{*}{ Materia } & \multirow{2}{*}{$\begin{array}{l}\text { Promedio de } \\
\text { notas }\end{array}$} & \multicolumn{3}{|c|}{ ¿Consideras que el Instituto te está preparando bien para la PAES? } \\
\hline & & Sí, muy bien & Más o menos & No me esta preprando \\
\hline \multirow[t]{4}{*}{ Matemáticas } & Menos de 5 & $61.1 \%$ & $22.2 \%$ & $16.7 \%$ \\
\hline & $5-6$ & $56.3 \%$ & $37.7 \%$ & $6.0 \%$ \\
\hline & $6.1-8$ & $52.8 \%$ & $38.0 \%$ & $9.2 \%$ \\
\hline & $8.1-10$ & $53.8 \%$ & $38.7 \%$ & $7.6 \%$ \\
\hline \multirow[t]{4}{*}{ Ciencias Naturales } & Menos de 5 & $58.3 \%$ & $25.0 \%$ & $16.7 \%$ \\
\hline & $5-6$ & $58.2 \%$ & $34.1 \%$ & $7.7 \%$ \\
\hline & $6.1-8$ & $54.2 \%$ & $38.5 \%$ & $7.4 \%$ \\
\hline & $8.1-10$ & $49.0 \%$ & $39.7 \%$ & $11.3 \%$ \\
\hline \multirow[t]{4}{*}{ Ciencias Sociales } & Menos de 5 & $14.3 \%$ & $57.1 \%$ & $28.6 \%$ \\
\hline & $5-6$ & $57.2 \%$ & $34.9 \%$ & $7.9 \%$ \\
\hline & $6.1-8$ & $55.9 \%$ & $37.1 \%$ & $7.0 \%$ \\
\hline & $8.1-10$ & $49.1 \%$ & $39.9 \%$ & $11.0 \%$ \\
\hline \multirow[t]{4}{*}{ Letras } & Menos de 5 & $47.1 \%$ & $35.3 \%$ & $17.6 \%$ \\
\hline & $5-6$ & $57.6 \%$ & $36.8 \%$ & $5.6 \%$ \\
\hline & $6.1-8$ & $56.8 \%$ & $35.8 \%$ & $7.4 \%$ \\
\hline & $8.1-10$ & $46.5 \%$ & $42.1 \%$ & $11.4 \%$ \\
\hline
\end{tabular}

Fuente: COP-UFG

Prueba Chi-cuadrado

De acuerdo con las pruebas chi-cuadrado en las asignaturas Matemática, Ciencias Naturales y Letras, el nivel de significancia es mayor a 0.05, por tanto, se rechaza la hipótesis de trabajo y se acepta la hipótesis nula, la cual establece que el promedio de notas en cada asignatura no depende de la preparación para la PAES de parte de la institución educativa.

Solamente en Ciencias Sociales se tiene un nivel de significancia inferior a 0.05 , por lo cual se acepta la hipótesis de trabajo, la cual indica que el promedio de notas en cada asignatura depende de la preparación para la PAES de parte de la institución educativa. 
En resumen, las pruebas chi-cuadrado muestran que en 3 de 4 asignaturas o el $75 \%$, el promedio de notas no depende de la preparación para la PAES de parte de la institución educativa. En el cuadro 3 se muestra como ejemplo, los valores obtenidos para Matemática.

Cuadro 3. Prueba chi-cuadrado para nota promedio en Matemática vs Preparación para la PAES de parte de la institución educativa.

Pruebas de chi-cuadrado

\begin{tabular}{lccc}
\hline & Valor & gl & Sig. asintótica (bilateral) \\
\hline Chi-cuadrado de Pearson & $5.645^{\circ}$ & 6 & .464 \\
\hline Razón de verosimilitudes & 5.662 & 6 & .462 \\
\hline Asociación lineal por lineal & .688 & 1 & .407 \\
\hline N. de casos válidos & 986 & & \\
\hline
\end{tabular}

a. 1 casillas (8.3\%) tienen una frecuencia esperada inferior a 5. La frecuencia mínima esperada es 1.50.

Fuente: elaboración propia a partir de la base de datos del estudio: "Evaluación de la calidad del sistema educativo público por estudiantes de educación media". Realizado por el Centro de Opinión Pública, Universidad Francisco Gavidia, 2013.

\section{Análisis de los indicadores: Promedio de notas vs Tiempo dedicado al estudio y tareas}

Tabla de contingencia

El cuadro 4 indica que en las cuatro asignaturas los porcentajes más altos corresponden a un tiempo de estudio diario entre una y tres horas, mientras que los porcentajes más bajos corresponden a un período de estudio de más de cuatro horas por día. Se deduce que la mayoría de estudiantes disponen de tiempo diariamente para dedicar al estudio y la minoría posiblemente por motivación personal dedica más de cuatro horas. Además, existe un porcentaje elevado de personas que dedica al estudio menos de 1 hora diaria, posiblemente debido a compromisos laborales.

Cuadro 4. Promedio de notas vs. Tiempo dedicado al estudio y tareas 


\begin{tabular}{|c|c|c|c|c|c|}
\hline \multicolumn{6}{|c|}{ PROMEDIO DE NOTAS CON TIEMPO PARA HACER LAS TAREAS } \\
\hline \multirow[t]{2}{*}{ Materia } & \multirow{2}{*}{$\begin{array}{l}\text { Promedio } \\
\text { de notas }\end{array}$} & \multicolumn{4}{|c|}{ ¿Cuánto tiempo dedicas diariamente a revisar lo visto en clase y a hacer tareas? } \\
\hline & & Menos de 1 hora & $1-2: 59$ horas & 3-4 horas & Más de 4:01 horas \\
\hline \multirow[t]{4}{*}{ Matemática } & Menos de 5 & $29.4 \%$ & $47.1 \%$ & $17.6 \%$ & $5.9 \%$ \\
\hline & $5-6$ & $31.2 \%$ & $57.6 \%$ & $9.2 \%$ & $2.0 \%$ \\
\hline & $6.1-8$ & $23.1 \%$ & $59.9 \%$ & $14.3 \%$ & $2.7 \%$ \\
\hline & $8.1-10$ & $23.5 \%$ & $63.0 \%$ & $9.2 \%$ & $4.2 \%$ \\
\hline \multirow[t]{4}{*}{ Ciencias Naturales } & Menos de 5 & $33.3 \%$ & $50.0 \%$ & $.0 \%$ & $16.7 \%$ \\
\hline & $5-6$ & $24.4 \%$ & $63.3 \%)$ & $10.6 \%$ & $1.7 \%$ \\
\hline & $6.1-8$ & $27.4 \%$ & $58.5 \%$ & $11.9 \%$ & $2.2 \%$ \\
\hline & $8.1-10$ & $20.1 \%$ & $58.8 \%$ & $16.7 \%$ & $4.4 \%$ \\
\hline \multirow[t]{4}{*}{ Ciencias Sociales } & Menos de 5 & $42.9 \%$ & $42.9 \%$ & $14.3 \%$ & $.0 \%$ \\
\hline & $5-6$ & $31.1 \%$ & $58.9 \%$ & $7.9 \%$ & $2.0 \%$ \\
\hline & $6.1-8$ & $23.9 \%$ & $61.1 \%$ & $12.3 \%$ & $2.7 \%$ \\
\hline & $8.1-10$ & $24.3 \%$ & $56.0 \%$ & $16.1 \%$ & $3.7 \%$ \\
\hline \multirow[t]{4}{*}{ Letras } & Menos de 5 & $31.3 \%$ & $50.0 \%$ & $18.8 \%$ & $.0 \%$ \\
\hline & $5-6$ & $31.3 \%$ & $55.6 \%$ & $11.1 \%$ & $2.1 \%$ \\
\hline & $6.1-8$ & $26.5 \%$ & $60.2 \%$ & $10.9 \%$ & $2.4 \%$ \\
\hline & $8.1-10$ & $18.5 \%$ & $62.2 \%$ & $16.1 \%$ & $3 . \%$ \\
\hline
\end{tabular}

Fuente: COP-UFG

Pruebas Chi-cuadrado

En las asignaturas: Matemática (cuadro 5 como ejemplo), Ciencias Sociales y Letras, el valor de significancia es mayor a 0.05 , por tanto se rechaza la hipótesis de trabajo y se acepta la hipótesis nula, la cual establece que el promedio de notas no depende del tiempo dedicado al estudio y tareas.

En Ciencias Naturales el valor de significancia es menor a 0.05 , por tanto se acepta la hipótesis de trabajo, la cual establece que el promedio de notas depende del tiempo dedicado al estudio y tareas.

En resumen, las pruebas chi-cuadrado muestran que en 3 de 4 asignaturas o el $75 \%$, el promedio de notas no depende del tiempo dedicado al estudio y tareas.

Cuadro 5. Prueba chi-cuadrado para nota promedio en Matemática vs Tiempo dedicado al estudio y tareas.

\begin{tabular}{lccc}
\hline & Valor & gl & Sig. asintótica (bilateral) \\
\hline Chi-cuadrado de Pearson & $12.891^{a}$ & 9 & .168 \\
\hline Razón de verosimilitudes & 12.687 & 9 & .177 \\
\hline Asociación lineal por lineal & 3.389 & 1 & .066 \\
\hline N. de casos válidos & 980 & & \\
\hline
\end{tabular}

a. 4 casillas (25.0\%) tienen una frecuencia esperada inferior a 5 . La frecuencia mínima esperada es .47.

Fuente: elaboración propia a partir de la base de datos del estudio: "Evaluación de la calidad del sistema educativo público por estudiantes de educación media". Realizado por el Centro de Opinión Pública, Universidad Francisco Gavidia, 2013. 


\section{Discusión y conclusión}

La evaluación periódica del sistema educativo público es indispensable para lograr alcanzar y mantener la calidad de la educación con estándares internacionales, obviamente la evaluación del desempeño docente es una parte de ese sistema. La PAES es solo uno de los parámetros a tomar en cuenta en la evaluación del sistema educativo, incluso en la evaluación del desempeño docente.

En la mayoría de los países existe la evaluación educativa nacional, menos en El Salvador; es del conocimiento público que la educación es de bajo nivel, no obstante, el Ministerio de Educación no realiza estudios para cualificar y cuantificar el bajo nivel en que se encuentra, si los haría, los indicadores se convirtieran en insumos para tomar medidas correctivas pertinentes e idóneas.

La evaluación del desempeño docente es beneficiosa para el profesor porque le permite tener conocimiento de lo acertado de su labor, en caso contrario asentirá a realizar los cambios apropiados; a los estudiantes porque incidirá en una mejora de su rendimiento escolar; a la institución educativa por la adquisición de prestigio académico por la excelencia de la planta docente; para el país porque se estarán formando futuros ciudadanos bien preparados con conocimientos, habilidades y valores; para la nación porque se incidirá en la formación de una nueva cultura, idiosincrasia, imaginario e identidad nacional.

Para obtener el buen desempeño docente, nos conduce a modificar la currícula de formación profesional, establecer políticas de ingreso a la carrera de profesorado, contrataciones de profesores por oposición y realización de pruebas de admisión, entre otras.

Se recomienda que el Estado a través del Ministerio de Educación retome la formación de profesores, debido a la comercialización de la educación que existe en El Salvador. Asimismo, modificar la Ley General de Educación y la Ley de la Carrera Docente para que la formación de maestros por parte del Estado y la evaluación del sistema educativo público adquieran rango de ley.

Antes de iniciar la evaluación del sistema educativo público, desarrollar una campaña publicitaria en los principales medios de comunicación y, capacitar a los profesores, para explicar en qué consiste y cuáles serán los alcances, de lo contrario, sería contraproducente, debido a la débil formación académica de los docentes, el atraso cultural, los intereses mercantiles de los propietarios de centros comerciales de educación y la polarización política del país.

La evaluación del desempeño docente contribuirá a establecer relaciones interpersonales horizontales de cooperación entre profesores, autoridades educativas, estudiantes y padres de familia, este clima social-educativo incidirá

La evaluación del desempeño docente en la educación pública 44. salvadoreña en la mejora del rendimiento escolar, del ambiente educativo y de la comunidad, por permitir desarrollar programas y proyectos ex aula que contribuirán a la formación de los estudiantes y en la mejora de la comunidad. 
En El Salvador urgen los cambios educativos, no hay que olvidar que la base fundamental de la democracia es la educación.

En este mismo orden, en el análisis estadístico descriptivo encontramos que el instrumento utilizado para evaluar el desempeño docente del sector público realizado por estudiantes, tiene algunas deficiencias las cuales se detallan a continuación:

1. Algunas preguntas no están bien redactadas, no son claras, ni precisas.

2. En algunas preguntas existen opciones de respuesta que no son pertinentes, son ambiguas, redundantes o similares entre ellas.

3. La mayoría de preguntas son superficiales y no se profundiza para investigar las causas de algún problema, hecho o situación dados.

4. Algunas preguntas no son pertinentes para investigar sobre una situación o problema específico.

5. El instrumento es limitado en relación a la cantidad de los aspectos de la calidad docente que evalúa.

Por las deficiencias antes señaladas, el cuestionario para evaluar la calidad del sistema educativo público, desde la perspectiva estudiantil, no fue elaborado con los patrones técnicos reflejados en los ítems pertinentes a los procesos, actores e infraestructura objetivo de la educación media.

El análisis chi cuadrado produjo como resultado que la nota promedio en las asignaturas no depende de la preparación para la PAES de parte de la institución educativa, ni tampoco depende del tiempo que el estudiante dedica al estudio y tareas. Por lógica el resultado esperado es la relación de dependencia entre los indicadores. Por tanto, se evidencia que el instrumento aplicado no fue diseñado adecuadamente, en lo que respecta a la evaluación de los indicadores considerados. Además, se debe tomar en cuenta que el análisis se basa en datos subjetivos, los cuales, resultan de la percepción de los estudiantes sobre su rendimiento académico y posiblemente dichos resultados no reflejan la realidad.

De acuerdo con la encuesta de opinión, los estudiantes evaluaron como satisfactorio el desempeño de los docentes del sector público. Este resultado se basa en la percepción que tienen los estudiantes, pero no significa que sea igual a la realidad.

\section{Referencias}

Bourdieu, P. (1997). Capital cultural, escuela y espacio social. Siglo XXI.

Gutiérrez, A. B. (2003). La educación como práctica social en la teoria de bourdieu: elementos de analisis a partir del caso de un conjunto de familias pobres de Córdoba, Argentina. Revista Complutense de Educación, 14(1), 115-131. http:// doi.org/-

Marín, J. G. (2010). Educación y reproducción cultural: el legado de Bourdieu. Práxis Educacional, 2(2). Recuperado a partir de http://periodicos.uesb.br/index.php/ praxis/article/view/380

Penagos, R. Á. (2009). Las relaciones entre la educación y la cultura en Pierre Bourdieu. 
Revista Colombiana de Sociología, 7(1), 9-26.

Sánchez, C. (2002, diciembre). En memoria de Pierre Bourdieu. Revista de la Universidad de Castilla-La Mancha, (12). Recuperado a partir de http://www.uclm.es/varios/ revistas/ docenciaeinvestigacion/numero2/carlossanchez.asp

Santos, M. (2011). Pierre Bourdieu: razón, escuela y disposición «escolástica». Presentado en XII Congreso Internacional de Teoría de la Educación, Barcelona. Recuperado a partir de http://www.cite2011.com.

Starkie, E. G. (2006). Evolución de la política educativa francesa: de la igualdad a la diversidad. Revista Complutense de Educación, 16(2), 415 - 438. http://doi.org/-

Tejedor, F. J. (2012). Evaluación del desempeño docente. RIEE. Revista lberoamericana de Evaluación Educativa, 319-327.

Zúñiga, L. C. M. (2009). Durkheim y Bourdieu: Reflexiones sobre educación. Revista Reflexiones, 88(1). Recuperado a partir de http://revistas.ucr.ac.cr/index.php/ reflexiones/article/view /11516 